\title{
Detecção de indícios de cartel: um estudo de caso para Belém/PA e Santarém/PA por meio de modelos de volatilidade
}

\author{
Detection of cartel evidence: a case study for Belém/PA and Santarém/PA using volatility models \\ Detección de pruebas de cárteles: un estudio de caso para Belém/PA y Santarém/PA utilizando \\ modelos de volatilidad
}

Recebido: 04/10/2021 | Revisado: 09/10/2021 | Aceito: 11/10/2021 | Publicado: 12/10/2021

\author{
Estevão Miguel Cardoso da Silva \\ ORCID: https://orcid.org/0000-0001-7543-1604 \\ Universidade Federal do Oeste do Pará, Brasil \\ E-mail: estevgremio@gmail.com \\ Brena do Nascimento Carvalho \\ ORCID: https://orcid.org/0000-0002-0660-8514 \\ Universidade do Estado do Amazonas, Brasil \\ E-mail: brenanc16@gmail.com \\ Zilda Joaquina Cohen Gama dos Santos \\ ORCID: https://orcid.org/0000-0002-5593-552X \\ Universidade Federal do Oeste do Pará, Brasil \\ E-mail: zilda.santos@ufopa.edu.br \\ Tarcísio da Costa Lobato \\ ORCID: https://orcid.org/0000-0002-2002-5622 \\ Universidade Federal do Oeste do Pará, País \\ E-mail: tarcisio.lobato@ufopa.edu.br
}

\begin{abstract}
Resumo
O objetivo deste artigo é detectar indício de cartel na aplicação de modelos de volatilidade em dados de preços de postos revendedores de gasolina dos municípios de Belém/PA e Santarém/PA. Cartéis são ações coordenadas entre firmas nas quais há acordos tácitos ou explícitos objetivando a coordenação de preços, quantidades ofertadas e/ou fatias de mercado, para maximização de lucro conjuntamente. Para detecção dos cartéis, serão aplicados modelos de volatilidade ARCH, GARCH, EGARCH e TGARCH. Os dados utilizados são os preços médios semanais de gasolina extraídos do portal oficial da Agência Nacional do Petróleo, Gás Natural e Biocombustíveis (ANP), no período de 2004 a 2020. Os resultados da equação para média não apontaram indícios de cartel, enquanto o modelo ARCH para variância detectou apenas em Belém. Não houve indícios de presença de choques assimétricos na série de Belém havendo apenas a ocorrência em Santarém. Conclui-se que a metodologia é útil para a detecção de cartéis de revendedores de gasolina.
\end{abstract}

Palavras-chave: Cartel; Modelos de volatilidade; Combustíveis.

\begin{abstract}
The aim of this paper is to detect evidence of cartel in the application of volatility models in price data of gas dealers in the municipalities of Belém/PA and Santarém/PA. Cartels are coordinated actions between firms in which there are tacit or explicit agreements aimed at price coordination, quantities offered and/or market slices, to maximize profit jointly. For the detection of cartels, arch, GARCH, EGARCH and TGARCH volatility models will be applied. The data used are the average weekly gasoline prices extracted from the official portal of the National Agency for Petroleum, Natural Gas and Biofuels (ANP), in the period from 2004 to 2020. The results of the equation for mean showed no indications of cartel, while the ARCH model for variance detected only in Belém. There were no indications of the presence of asymmetric shocks in the Belém series, with only the occurrence in Santarém. It is concluded that the methodology is useful for the detection of cartels of gasoline dealers.
\end{abstract}

Keywords: Cartel; Volatility models; Fuels.

\section{Resumen}

El objetivo de este artículo es detectar evidencias de cártel en la aplicación de modelos de volatilidad en los datos de precios de las estaciones de los distribuidores de gasoline municipios de Belém/PA y Santarém/PA. Los cárteles son acciones coordinadas entre empresas en las que existen acuerdos tácitos o explícitos destinados a la coordinación de precios, cantidades ofrecidas y/o rebanadas de mercado, para maximizar las ganancias juntos. Para la detección de cárteles se aplicarán los modelos de volatilidad arch, GARCH, EGARCH y TGARCH. Los datos utilizados son los precios promedio semanales de la gasolina extraídos del portal oficial de la Agencia Nacional del Petróleo, Gas 
Natural y Biocombustibles (ANP), de 2004 a 2020. Los resultados de la ecuación para la media no mostraron indicios de cártel, mientras que el modelo ARCH para la varianza se detectó solo en Belém. No hubo indicios de la presencia de choques asimétricos en la serie de Belém, con sólo la ocurrencia en Santarém. Se concluye que la metodología es útil para la detección de cárteles de distribuidores de gasolina.

Palabras clave: Cartel; Modelos de volatilidade; Combustibles.

\section{Introdução}

Compreender as dinâmicas e comportamentos dos agentes econômicos na sociedade é uma das principais contribuições que a Ciência Econômica tem entregado ao campo do conhecimento científico ao longo de vários anos. Nesse sentido, esta tarefa pode ser definida como o estudo dos mais diversos mercados que se configuram de diferentes maneiras dadas as condições e relações existentes entre os agentes que o integram.

A partir disso, desses agentes (firmas) decidem realizar acordos explícitos (cartéis) com o intuito, sobretudo, de superar as condições de concorrência de mercado, e assim extrair resultados que não seriam possíveis sem a formação do acordo. Assim, surge a necessidade de formas de detectar este tipo de comportamento, pois diversos desses acordos são mantidos com extrema cautela pelas firmas e não transparecem muita das vezes aos consumidores ou às autoridades legais, permanecendo em grande parte das vezes por anos reduzindo o bem-estar dos consumidores e prejudicando a concorrência.

No Brasil, atualmente, um dos principais esforços despendidos pelo Sistema Brasileiro de Defesa da Concorrência (SBDC) está em combater as frequentes associações colusivas entre firmas no mercado de combustíveis brasileiro, as quais incorrem tanto no varejo (postos de combustíveis) quanto no atacado (setor de distribuição), prejudicando a concorrência ao retirar dos consumidores sua capacidade de escolha no mercado. O setor de varejo de postos de combustíveis é responsável por frequentes denúncias que relatam possíveis associações ilegais entre firmas prejudicando a concorrência.

Nesse sentido, no Brasil cartéis e ações contra a concorrência são considerados crimes de acordo Lei n 12.529 , de 30 de novembro de 2011. Sua aplicação na esfera administrativa, é realizada por dois órgãos, que compõem o chamado Sistema Brasileiro de Defesa da Concorrência (SBDC): a Secretaria de Acompanhamento Econômico do Ministério da Fazenda (SEAE/MF) e o Conselho Administrativo de Defesa Econômica (CADE), autarquia vinculada ao Ministério da Justiça (Voronkoff, 2014). Contudo, a formação de cartel no mercado de combustível é facilitado por diversas características, como por exemplo: cadeias de produção, distribuição e comercialização fortemente integradas; diversas associações de postos nos municípios; barreiras à entrada no setor; conduta criminosa contra aqueles que não aderem ao cartel; dependência espacial dos combustíveis demandados; grande dificuldade em identificar os cartéis; facilidade de organização e fiscalização entre as firmas; homogeneidade dos combustíveis; e leniência do poder público (Silva, 2016).

A literatura econômica sobre métodos e estratégias de detecção de cartéis cresceu bastante no decorrer dos anos com o suporte teórico da economia industrial e dos estudos empíricos sobre casos que ocorreram e puderam ser investigados. Reconhecidamente na literatura os métodos de detecção de cartéis recebem o nome de screens. De acordo com Abrantes-Metz e Bajari (2009), os screens possuem a função de identificar indústrias que possuem problemas e aspectos que comprometam a concorrência, e dentre essas indústrias quais são as firmas que podem estar envolvidas em um acordo de cartel. Os autores também destacam que há muitos usos para os screens, tais como: ferramentas de defesa para acusados como prova de não colusão; implementação de programas de leniência; estimação de danos econômicos e de multas.

Os screens não provam o acordo de cartel entre as firmas, servindo apenas para diferenciar os resultados que seriam obtidos em um mercado competitivo em ralação a um mercado com a presença de um cartel, indicando assim onde a autoridade legal pode imprimir maiores esforços para combater esse tipo de acordo. Portanto, mesmo que os screens forneçam apenas evidências econômicas de um cartel, eles podem fornecer provas lógicas que possuam o mesmo peso de provas legais (Buccirossi, 2006; Abrantes-Metz; Bajari, 2009). 
Nesse sentido, pesquisar o setor de combustíveis torna-se relevante para entender como tais cartéis operam, quais são suas características e como detectá-los. Somado a isto, o CADE necessita cada vez mais de estudos de detecção de cartéis para subsidiar as investigações de denúncias de paralelismo de preço entre firmas. Assim, a função da literatura econômica é fornecer evidências para a ação investigativa dos órgãos que atuam no combate a cartéis. No estado do Pará, até o presente momento, a literatura econômica é escassa no tratamento deste tema e por este motivo se pretende analisar os municípios de Belém e Santarém para que a mesma possa ser complementada e fortalecida com as novas evidencias a partir da aplicação dos modelos e da análise econômica apresentada.

De acordo com o Instituto Brasileiro de Geografia e Estatística (IBGE), O estado do Pará concentra 2.229.234 de veículos que utilizam alguma forma de combustível líquido para funcionamento. Desse total, 486.052 estão concentrados no município de Belém, e 113.088 estão no município de Santarém (IBGE, 2021). Relacionado a isto, o anuário estatístico de 2020 realizado pela ANP contabilizou um total de 1.214 postos de revenda de combustíveis no estado do Pará, sendo que de acordo com o programa Levantamento de Preços de Combustíveis (LPC) realizado pela Agência Nacional do Petróleo, Gás Natural e Biocombustíveis (ANP), a pesquisa semanal mais recente em 2021 contava com o registro de 20 postos do município de Belém e 12 do município de Santarém (ANP, 2020).

Dessa maneira, este estudo tem como objetivo analisar as séries de preços da gasolina $\mathrm{C}$ dos municípios de Belém/PA e Santarém/PA em busca de possíveis indícios de cartéis entre firmas no varejo aplicando modelos econométricos de volatilidade. Para isto, serão aplicados modelos de volatilidade ARCH (Autoregressive Conditional Heteroscedasticity), GARCH (Generalized Autoregressive Conditional Heteroskedasticity), EGARCH (Exponential Generalized Autoregressive Conditional Heteroskedastic) e TGARCH (Threshold Generalized Autoregressive Conditional Heteroskedasticity) nas séries de preços da gasolina $\mathrm{C}$ dos municípios de Belém/PA e Santarém/PA, utilizando os dados semanais fornecidos pelo portal oficial ANP. Serão realizadas análises na variância e média das séries para identificar indícios de cartel.

Por fim, este trabalho está dividido em cinco seções. A seção 1 apresenta a introdução do estudo. A seção 2 apresenta o referencial teórico. A seção 3 traz a descrição dos procedimentos metodológicos, a literatura acerca de métodos de detecção de carteis por meio de ferramentas estatísticas, bem como apresenta os modelos econométricos que serão utilizados. A seção 4 contém os resultados da aplicação dos modelos. E por último, a seção 5 traz as considerações finais deste estudo.

\section{Revisão da Literatura}

A respeito das formas de detecção de cartéis, Harrington (2006) e Grout e Sonderegger (2006) apresentam, respectivamente, perspectivas de análise baseadas no comportamento (behavioral approach) e na estrutura das firmas (Structural Approaches). Em ambas as abordagens a análise do comportamento dos preços é elemento fundamental, visto que a pode armazenar as informações que permitem ser analisadas e testadas para a verificação de práticas anticompetitivas entre firmas. Harrington (2006), a partir da análise comportamental define marcadores (Collusive Marker) de preço (Price Markers) e de quantidade (Quantity Markers), que visam aprimorar a triagem (sceening) em casos em que há suspeita de acordos colusivos entre firmas. O segundo modelo de abordagem para a detecção de cartéis segue a linha estrutural (Structural Approaches). Nesta abordagem, Grout e Sonderegger (2006) apontam para as estruturas e características das firmas, dos bens e do mercado que facilitam e tornam a existência de cartéis mais provável do ponto de vista estrutural.

Ademais, de acordo com Silva (2016) não necessariamente tais características apontadas na abordagem estrutural são indícios de cartéis, uma vez que pode haver um efeito inverso no qual tais aspectos estejam sendo produzidos por um cartel. Desse modo, a abordagem estruturalista aponta as estruturas e características de mercado que de certa forma dão margem para a formação de cartéis, sem de fato apontar os indícios que levariam a identificar um cartel. Nisto a abordagem comete o erro de atestar falsos casos como positivos em testes que façam o seu uso (Harrington, 2005). 
Cartéis são ações coordenadas entre firmas nas quais há acordos tácitos ou explícitos objetivando a coordenação de preços, quantidades ofertadas e/ou fatias de mercado, para maximização de lucro conjuntamente. Cartéis em que há um acordo tácito entre as firmas sem uma definição formal de preços, quantidades ou fatias de mercados, são considerados cartéis tácitos. Apesar da distinção feita entre as duas formas de acordo, em grande parte das vezes os danos ao bem-estar e a liberdade de escolha dos consumidores são os mesmos (Harrington, 2005).

De acordo com Harrington (2012), um resultado econômico obtido a partir da formação de preços supra competitivos, sem que houvesse sido estabelecido um acordo formal entre as firmas configura um acordo de cartel tácito. De outro modo, quando há a deliberação de um acordo formal entre as firmas em prol de coordenar preços, quantidades e/ou fatias de mercado, esse tipo de associação recebe o nome de cartel explícito (Harrington, 2005).

Dessa maneira, a distinção entre as formas de associações de firmas em cartel é fundamental para o entendimento do caráter jurídico de tais associações, haja vista que o modelo de cartel considerado ilegal em diversos países é o modelo no qual há um acordo anticompetitivo explícito entre as firmas. Harrington (2021) indica três razões que fazem com que cartéis existam e, por vezes, sejam bem sucedidos dentro de certos mercados, sejam elas: (i) mercados com bens muito similares e competição por preços acirrada; (ii) quando um conjunto de firmas (dirigentes) percebem a competição como um obstáculo para a extração de lucros suficientes elas tem o incentivo para entrar em um acordo; e (iii) as muitas maneiras de se realizar acordos de restrição da concorrência, não necessitando de elevada sofisticação nas estratégias para que isso seja feito.

Nesse sentido, diversos estudos compõe a literatura que trata de cartéis e sobre o comportamento e as formas como as firmas estabelecem acordos para burlar a concorrência e extrair ganhos superiores àqueles que se teriam sob um regime competitivo. Os estudos que utilizam modelos de volatilidade para detecção de indícios de cartéis foram bastante aprimorados ao longo dos anos. Atualmente os modelos são capazes de detectar com precisão as dinâmicas dos preços sob regimes competitivos e de carteis, contribuindo para que as autoridades antitruste ajam com precisão e eficiência no combate e na investigação desse tipo de atividade.

Abrantes-Metz et al. (2006) tomando como base os preços médios semanais do mercado de varejo de gasolina durante os anos de 1996 a 2002, desenvolveram um trabalho sobre cartéis no mercado varejista de combustíveis e observaram as variâncias dos preços dos combustíveis a partir da criação de setores estratégicos com base em rotas de deslocamento populacional e localização dos postos. O trabalho foi baseado no caso de cartéis de licitações (bid-rigging) em que empresas de alimentos marítimos fraudavam a concorrência. A conclusão dos autores foi que na análise dos preços dos postos de combustíveis o filtro quando aplicado em Louisville nos Estados Unidos não encontrou evidências de comportamento colusivo nas áreas analisadas.

A esse respeito, Vasconcelos e Vasconcelos (2008) utilizando a série de preços de São Paulo, Florianópolis e Recife, fizeram testes com modelos ARCH e GARCH para analisar os preços médios e a variância das séries sob um período hipotético de cartel. Os modelos confirmaram a hipótese de preços superiores nas séries de preços de São Paulo e Recife, e a hipótese de redução da variância se confirmou apenas em Recife. Os autores concluíram que a metodologia empregada é adequada para a detecção de cartéis.

Bolotova et al. (2008) fizeram a aplicação de dois modelos, ARCH e GARCH, para um período de cartel e outro competitivo, analisando o comportamento da série de preços dos cartéis descobertos na indústria de Lisina e Ácido Cítrico. Os resultados dos modelos revelaram que durante o período de conspiração as firmas elevaram os preços de 9 a 25 centavos por libra. Os autores também encontraram que a variância dos preços da série da Lisina foi menor durante o período de conspiração do que durante o período sem conspiração, enquanto a da série do Ácido Cítrico foi maior do que no período de não conspiração. Os autores ainda recomendam o uso da metodologia para a detecção de cartéis. 
Silveira et al. (2019) realizaram testes utilizando duas metodologias, quais sejam, Markov-Switching GARCH (MSGARCH) Models e Local Gaussian Correlation (LGC) approach, para analisar o comportamento anticompetitivo no varejo de combustíveis tomando como benchmark o caso dos cartéis de Brasília. De acordo com os autores, o modelo MS-GARCH identifica o comportamento de cartel entre firmas a partir da análise da volatilidade dos preços e da dispersão dos preços. Os resultados encontrados pelos autores indicaram que o modelo LGC pode apresentar probabilidade viesada e problemas de identificação do comportamento do cartel ao longo do tempo. Já o modelo MS-GARCH apresentou melhor performance pois forneceu respostas mais acuradas para a detecção de cartéis.

A discussão acerca da presença de assimetria na transmissão de preços do atacado para o varejo no setor de combustíveis foi também incrementada com o estudo de Salvini et al. (2017), no qual os autores estudaram o mercado de combustíveis do estado de São Paulo para entender a dinâmica e o comportamento dos preços tanto no atacado quanto no varejo. Os autores utilizaram as médias mensais dos preços da gasolina comum e do etanol hidratado nos níveis de distribuição e revenda para o período de novembro de 2002 a abril de 2015 empregando Modelos de Correção de Erros. Os resultados evidenciaram que há presença de assimetria positiva na transmissão dos preços dos dois produtos nos dois setores no curto prazo. Dessa forma, os autores concluíram que aumentos nos preços no atacado elevam com maior intensidade os preços ao consumidor, em comparação a decréscimos provocados por choques negativos nos preços de distribuição.

\section{Metodologia}

\subsection{Fontes de informação}

A base de dados foi extraída do site oficial da ANP, a qual contém os preços médios semanais da gasolina C, obtidos por meio do programa Levantamento de Preços de Combustíveis (LPC) realizado pela própria ANP. Dentro da base de dados total fornecida pela ANP 19 municípios do estado do Pará possuem o monitoramento de preços pela ANP, entretanto serão considerados apenas os municípios de Belém/PA e Santarém/PA, pois são os que apresentam amostragem de postos de revenda e observações de preços semanais adequados viabilizando a aplicação dos modelos. Portanto, a extensão total da série possui 811 observações de preços semanais totais para a gasolina C durante o período que vai de 2004 a 2020. Este período corresponde a série global, e foi tomado como ponto de partida para a verificação de períodos intermediários onde os preços apresentem comportamento próximo aos de um cartel.

Adicionalmente, a análise da descritiva dos dados, bem como todos os gráficos presentes neste estudo foram feitos com o software Python. O ajuste e a análise dos modelos econométricos foram feitos com o uso do software Eviews Statistic nas versões 10 e 12 para estudante.

\subsection{Modelos de volatilidade}

Sobre a modelagem para a volatilidade e média da série de preços da gasolina $\mathrm{C}$, há evidências que já corroboram a boa adequação dos modelos ARCH, desenvolvido por Engle (1982), e GARCH, desenvolvido por Bollerslev (1986) para análise do comportamento de preços para casos de cartel. Isto é confirmado por Bolotova et al. (2008) ao apontar duas vantagens dos modelos, quais sejam: (i) boa resposta na captação dos períodos de baixa volatilidade (variância) e (ii) uma baixa quantidade de dados para execução do modelo. Os autores ainda ressaltam que os modelos ARCH e GARCH permitem também a modelagem do comportamento da média e da variância da série simultaneamente. Ademais, observar estes elementos simultaneamente permite identificar possíveis cartéis que ao invés de elevarem seus preços, os homogeneízam gerando lucros adicionais.

Os modelos ARCH-GARCH captam simetricamente os retornos. Dessa forma, a modelagem fica restrita aos efeitos simétricos dos retornos na modelagem da série, além da necessidade de os coeficientes do modelo serem positivos (Marques, 
2017). Assim, algumas extensões para esses modelos surgiram objetivando complementá-los e serão usadas neste trabalho, sejam elas: o modelo EGARCH e o modelo TGARCH.

O modelo EGARCH foi proposto por Nelson (1991), e sua proposta era captar os choques assimétricos dos retornos na volatilidade da série (Morettin, 2017). Já o modelo TGARCH foi desenvolvido por Zakoian (1994) e Glosten et al. (1993) capta os choques assimétricos de good news e bad news na volatilidade da série.

\subsubsection{Modelo ARCH}

Para um modelo $\mathrm{ARCH}$, ainda que os retornos na equação 1 são não correlacionados, entretanto suas variâncias são dependentes das observações de retornos passadas por uma função quadrática (Greene, 2000; Morettin, 2017).

$$
\begin{aligned}
\varepsilon_{\mathrm{t}} & =v_{\mathrm{t}} \sqrt{h_{\mathrm{t}}} \\
h_{\mathrm{t}} & =\alpha_{0}+\beta_{1} \varepsilon_{\mathrm{t}-1}^{2}+\cdots+\beta_{\mathrm{i}} \varepsilon_{\mathrm{t}-\mathrm{i}}^{2}
\end{aligned}
$$

onde $\varepsilon_{\mathrm{t}}$ é i.i.d. $(0,1), a_{0}>0,1 \geq \beta_{1} \geq 0, i>0$, e é condicionalmente heterocedástico com relação a $\varepsilon_{\mathrm{t}-\mathrm{i}}$.

\subsubsection{Modelo GARCH}

O modelo GARCH é um modelo mais parcimonioso do que o modelo ARCH por requerer uma baixa quantidade inferior de parâmetros para estimar a volatilidade da série. Dessa forma, a equação 2 apresenta a estrutura do modelo GARCH $(p, q)$ (MORETTIN, 2017).

$$
\begin{aligned}
& \varepsilon_{\mathrm{t}} \mid \Psi_{\mathrm{t}-1} \sim N\left(0, h_{\mathrm{t}}\right) \\
& \varepsilon_{\mathrm{t}}=v_{\mathrm{t}} \sqrt{h_{\mathrm{t}}}
\end{aligned}
$$

$$
h_{\mathrm{t}}=\alpha_{0}+\sum_{i=1}^{p} \beta_{i} \varepsilon_{t-i}^{2}+\sum_{i=1}^{q} \alpha_{i} h_{t-i}
$$

onde $\varepsilon_{\mathrm{t}}$ i.i.d. $(0,1), \alpha_{0}>0, \alpha_{\mathrm{i}} \geq 0, \beta_{\mathrm{i}} \geq 0, \sum_{\mathrm{i}=1}^{k}\left(\alpha_{\mathrm{i}}+\beta_{\mathrm{i}}\right)<1, k=\max \left(p_{s} q\right)$.

\subsubsection{Modelo EGARCH}

O modelo EGARCH é capaz de capturar os choques assimétricos na volatilidade da série (ENDERS, 2008; MORETTIN, 2017). Dessa forma, a equação 3 traz a estrutura do modelo EGARCH.

$$
\begin{aligned}
\varepsilon_{\mathrm{t}} & =v_{\mathrm{t}} \sqrt{h_{\mathrm{t}}} \\
\ln \left(h_{\mathrm{t}}\right) & =\alpha_{0}+\beta_{1}\left(\frac{\varepsilon_{\mathrm{t}-1}}{h_{\mathrm{t}-1}^{0.15}}\right)+\lambda_{1}\left|\frac{\varepsilon_{\mathrm{t}-1}}{h_{\mathrm{t}-1}^{0.5}}\right|+\alpha_{1} \ln \left(h_{\mathrm{t}-1}\right)
\end{aligned}
$$

onde embora $h_{\mathrm{t}}$ não possa assumir valores negativos, os coeficientes da equação podem. Dessa forma, o modelo EGARCH permite a captura de leaverage effects (efeitos de alavancagem) derivados dos choques na variância. Assim, se $\varepsilon_{\mathrm{t}-1} / h_{\mathrm{t}-1}^{0.5}$ for positivo, o efeito dos choques no log da variância condicional serão $\beta_{1}+\lambda_{1}$. Caso contrário, se $\varepsilon_{t-1} / h_{t-1}^{0.5}$ for negativo, o efeito dos choques no log da variância condicional será dado por $-\beta_{1}+\lambda_{1}$.

\subsubsection{Modelo TGARCH}

O modelo TGARCH também capta a assimetria dos choques dos retornos na volatilidade da série, sendo que a sua volatilidade funcional é conforme a equação 4 (ENDERS, 2008; MORETTIN, 2017).

$$
h_{\mathrm{t}}=\alpha_{0}+\beta_{1} \varepsilon_{\mathrm{t}-1}^{2}+\lambda_{1} \kappa_{\mathrm{t}-1} \varepsilon_{\mathrm{t}-1}^{2}+\alpha_{1} h_{\mathrm{t}-1}
$$


em que $\kappa_{t-1}$ é uma variável dummy que assume valores iguais a 1 se $\varepsilon_{t-1} \leqslant 0$, e 0 , se $\varepsilon_{t-1} \geq 0$. Nesse sentido, $\varepsilon_{t-k} \geq 0$, faz com que um choque de $\varepsilon_{\mathrm{t}-1}$ em $h_{\mathrm{t}}$ seja dado por $\alpha_{1} \varepsilon_{\mathrm{t}-1}^{2}$. Noutro caso quando, $\varepsilon_{\mathrm{t}-1}<0$, e $\kappa_{\mathrm{t}-1}=1$, o efeito de $\varepsilon_{\mathrm{t}-1}$ em $h_{\mathrm{t}}$ será dado por $\left(\beta_{1}+\lambda_{1}\right) \varepsilon_{t-1}^{2}$. Ainda, se $\lambda_{1}>0$, choques negativos terão maior efeito na volatilidade do que choques positivos, e somado a isto, se verificada a significância do coeficiente $\lambda_{1}$, pode-se concluir que o conjunto de dados possui efeito threshold.

\subsection{Procedimentos para a aplicação dos modelos}

Para a aplicação dos modelos nas séries dos municípios foi tomada como referência a metodologia contida em Bolotova, et al. (2008) e Vasconcelos e Vasconcelos (2008), onde os autores ao modelar a variância e a média das séries por meio dos modelos econométricos selecionados, utilizaram uma variável dummy, $d_{t}$, assumindo valores 1 para os períodos da série onde ocorreram os cartéis, e 0 para os períodos da série selecionados como pré e pós cartel nos quais foi considerada a ausência de cartel. Ademais, o primeiro passo para a modelagem da série foi extrair os retornos, $R_{t}$, das séries de preços dos dois municípios por meio da diferenciação dos $\operatorname{logs}$ da série. Este procedimento é simplificadamente a logaritmização da diferença dos preços de uma semana (anterior) para os da semana posterior, como mostra a equação 5.

$$
R_{\mathrm{t}}=\log \left(\frac{\mathrm{p}_{\mathrm{L}}}{\mathrm{p}_{\mathrm{L}-\mathrm{I}}}\right)
$$

Dessa forma, para a aplicação dos modelos, foram escolhidos dois períodos hipotéticos de cartel em cada uma das séries como base a metodologia para detecção de indícios de cartéis contida em Pedra et al. (2010), Ragazzo e Silva (2006) e Carrijo (2019). Assim, é justa a escolha dos períodos selecionados pois foram os que excederam o limite da média da margem de revenda mais um incremento de 10\%, como nos gráficos 2 e 4, e o Coeficiente de Variação dos Preços de Revenda ${ }^{1}$ (CVPR) nos dois períodos apresentou redução em comparação com o restante das séries como evidenciado na Tabela 1.

Tabela 1. Períodos selecionados para a análise e estatísticas descritivas das séries.

\begin{tabular}{|c|c|c|c|c|c|c|}
\hline Município & Período & $N$ & $\begin{array}{c}\text { Média dos } \\
\text { Preços R\$/I } \\
\end{array}$ & Variância & $\begin{array}{c}\text { Média da Margem } \\
\text { de revenda }(\%)\end{array}$ & $C V P R^{*}$ \\
\hline \multirow{4}{*}{ Santarém } & Série completa & 812 & 3.29 & 0.58 & 0.14 & 0.01 \\
\hline & Pré-Cartel 16/01/2010 - 15/01/2011 & 53 & 2.79 & 0.00 & 0.14 & 0.01 \\
\hline & Cartel 16/01/2011 - 11/11/2017 & 343 & 3.40 & 0.26 & 0.16 & 0.01 \\
\hline & Pós-Cartel 19/11/2017 - 17/11/2018 & 49 & 4.57 & 0.03 & 0.13 & 0.02 \\
\hline \multirow{4}{*}{ Belém } & Série completa & 869 & 3.17 & 0.54 & 0.11 & 0.03 \\
\hline & Pré-Cartel 10/12/2006 - 08/12/2007 & 52 & 2.52 & 0.00 & 0.10 & 0.05 \\
\hline & Cartel 09/12/2007 - 11/07/2010 & 133 & 2.72 & 0.00 & 0.15 & 0.02 \\
\hline & Pós-Cartel 18/07/2010 - 16/07/2011 & 51 & 2.70 & 0.00 & 0.12 & 0.04 \\
\hline
\end{tabular}

*Coeficiente de Variação dos Preços de Revenda.

Fonte: Autores, a partir dos dados da pesquisa.

\subsubsection{Período de cartel hipotético em Santarém/PA}

O período selecionado para a série de Santarém/PA, vai de 16/01/2011 a 11/11/2017, como mostra a Figura 1. Observando a análise das estatísticas descritivas da série contidas na Tabela 1, fica evidente que há uma elevação na média dos

\footnotetext{
${ }^{1} \mathrm{O}$ coeficiente CVPR é a medida formada através do quociente entre o desvio-padrão e a média da série de preços de revenda servindo para analisar o grau de dispersão dos preços da série, e é dada seguinte fórmula, CVPR $=\sigma / \bar{x}$ (Pedra et al., 2010).
} 
preços no período de cartel, $\mathrm{R} \$ / \mathrm{L} 3,40$, em comparação tanto com a média da série global, $\mathrm{R} \$ / \mathrm{L} 3,29$, quanto ao período preestabelecido do cartel, $\mathrm{R} \$ / \mathrm{L} 2,79$.

Figura 1. Série de preços da gasolina C de Santarém/PA com período hipotético de cartel.

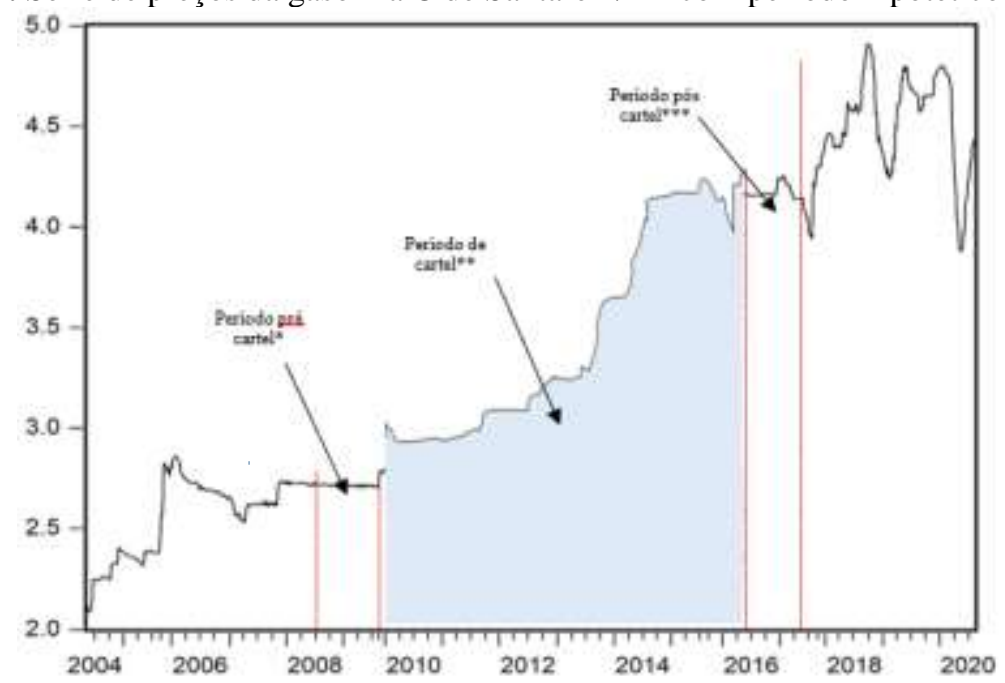

*Pré-cartel 16/01/2010 - 15/01/2011. **Cartel 16/01/2011 - 11/11/2017. ***Pós Cartel 19/11/2017 - 17/11/2018. Fonte: Autores, a partir dos dados da pesquisa.

Somado a isto, pode-se observar também que há elevação da margem percentual de revenda pelos postos no período de cartel, passando de 0,142\% no período pré-cartel para 0,159\% no período de cartel conforme exibe a Figura 2. Convém ainda destacar que nas estatísticas descritivas não há redução nem na variância da série nem no coeficiente CVPR. Tal fato poderia ser explicado por fatores como postos dissidentes ao acordo de cartel ou guerras de preços envolvendo os integrantes do cartel durante o período.

Figura 2. Margem percentual de revenda da gasolina C de Santarém/PA.

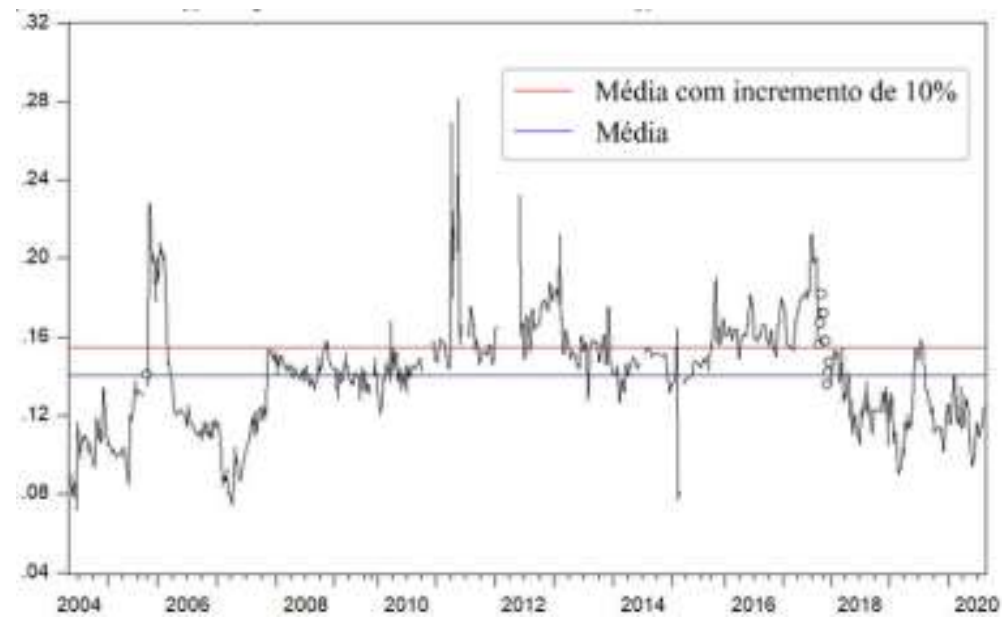

Fonte: Autores, a partir dos dados da pesquisa.

\subsubsection{Período de cartel hipotético em Belém/PA}

O período de cartel suposto para a série de Belém/PA vai de 09/12/2007 a 11/07/2010 e pode ser visto na Figura 3. Ao total são 133 semanas nas quais o preço médio foi de $\mathrm{R} \$ / \mathrm{L} 2,72$. O preço médio do período de cartel é inferior ao preço médio da série completa, $\mathrm{R} \$ / \mathrm{L} 3,17$, e superior ao do período preestabelecido do cartel, $\mathrm{R} \$ / \mathrm{L} 2,52$. O preço do período considerado como pós rompimento do cartel é $\mathrm{R} \$ / \mathrm{L} 2,70$, menor do que durante o cartel, porém não representa uma redução 
nos mesmos níveis do acréscimo ocorrido na transição do período pré-cartel para o período de cartel. Este fato pode ser entendido como uma persistência dos níveis de preços colusivos como uma herança do cartel para os consumidores, ou como um efeito de um acordo implícito entre os postos para a manutenção dos níveis de preços em colusivos mesmo após o rompimento do cartel.

Figura 3. Série de preços da gasolina $\mathrm{C}$ de Belém/PA com período hipotético de cartel.

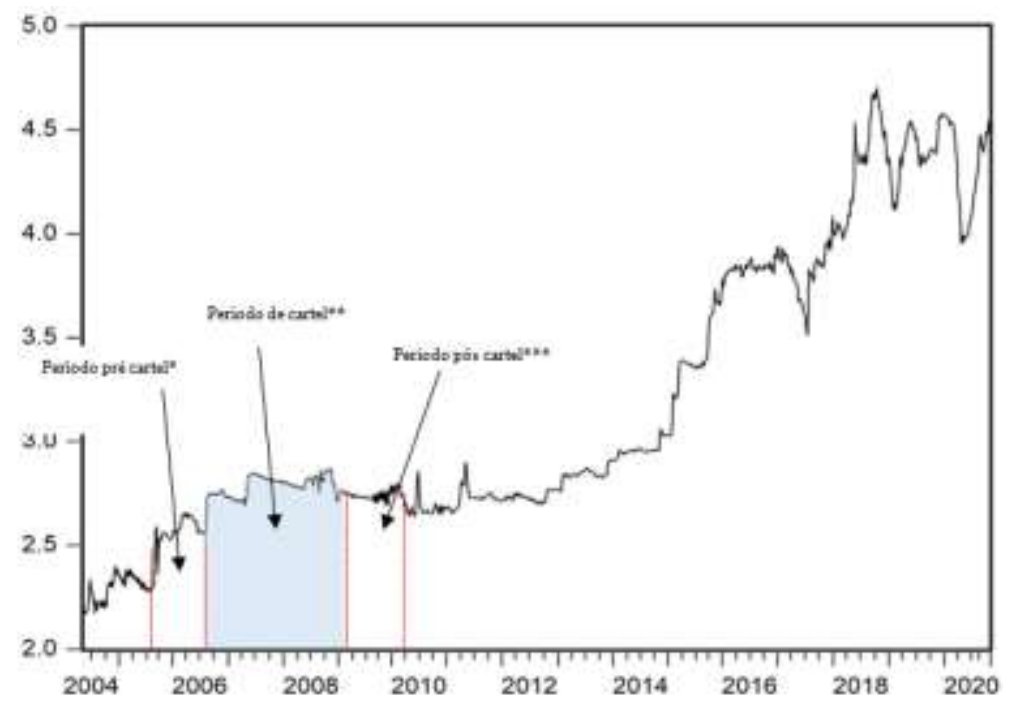

*Pré-cartel 10/12/2006 - 08/12/2007. **Cartel 09/12/2007 - 11/07/2010. ***Pós cartel 18/07/2010 - 16/07/2011. Fonte: Autores, a partir dos dados da pesquisa.

A média da margem de revenda dos postos no período de cartel, 15\%, foi superior à média da série completa, 11,4\%, superior à do período pré-cartel, 10,4\%, e inferior à média do período pós cartel, 11,8\%, no qual foi suposto haver o rompimento do acordo de cartel. O Figura 4 mostra esta dinâmica. Em acréscimo, houve também redução nos indicadores de dispersão dos preços no período de cartel, diferindo dos indicadores dos períodos não colusivos que apresentaram valores superiores.

É válido destacar que este estudo leva em consideração apenas o preço de compra dos combustíveis junto às distribuidoras como sendo o principal fator de custo dos postos na hora de definir seus preços. Assim, mesmo que haja outros custos como os de logística, armazenamento, administrativos etc., os custos de aquisição junto as distribuidoras ainda são os principais determinantes dos preços ofertados pelos postos. 
Figura 4. Margem percentual de revenda da gasolina $\mathrm{C}$ de Belém/PA.

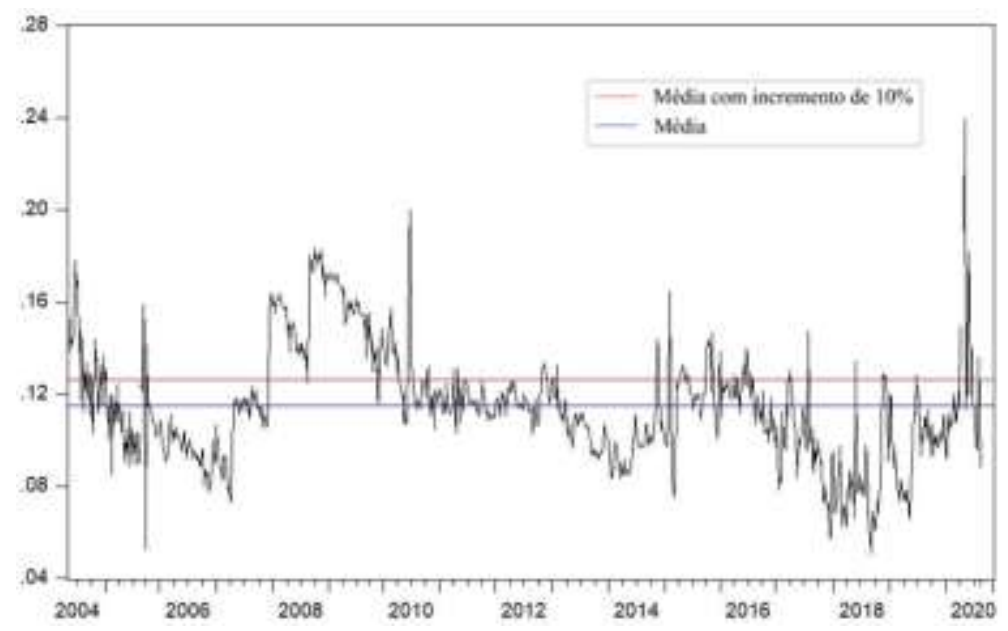

Fonte: Autores, a partir dos dados da pesquisa.

Os modelos com adição da variável dummy, $d_{t}$, para a média na equação (6), e para a variância nas equações (7), (8), (9) e (10), representando, respectivamente, os modelos ARCH, GARCH, EGARCH e TGARCH, ficam da seguinte forma na Tabela 2 .

Tabela 2. Modelos com a inclusão da variável dummy.

\begin{tabular}{lll}
\hline AR & $\begin{array}{l}Y_{t}=\alpha_{0}+\theta_{1} Y_{t-1}+\varphi_{1} d_{t}+\varepsilon_{t} \\
(6)\end{array}$ \\
\hline ARCH & $h_{t}=\alpha_{0}+\beta_{1} \varepsilon_{t-1}^{2}+\varphi_{1} d_{t}$ \\
\hline GARCH & $h_{t}=\alpha_{0}+\sum_{i=1}^{q} \beta_{i} \varepsilon_{t-1}^{2}+\sum_{i=1}^{p} \alpha_{i} h_{t-1}+\varphi_{1} d_{t}$ \\
\hline EGARCH & $\ln \left(h_{t}\right)=\alpha_{0}+\beta_{1}\left(\frac{\varepsilon_{t-1}}{h_{1-1}^{05}}\right)+\lambda_{1}\left|\frac{\varepsilon_{1-1}}{h_{1-1}^{05}}\right|+\alpha_{1} \ln \left(h_{t-1}\right)+\varphi_{1} d_{t}$ \\
\hline TGARCH & $h_{t}=\alpha_{0}+\beta_{1} \varepsilon_{t-1}^{2}+\lambda_{1} K_{t-1} \varepsilon_{t-1}^{2}+\alpha_{1} h_{t-1}+\varphi_{1} d_{t}$
\end{tabular}

Fonte: Autores, a partir dos dados da pesquisa.

\section{Resultados}

A partir da extração dos retornos pela logaritmização e diferenciação das séries conforme a equação 5 , foi testada a estacionariedade de ambas as séries por meio dos testes augmented Dickey-Fuller (ADF) desenvolvido por Dickey e Fuller (1979) e Kwiatkowski-Phillips-Schmidt-Shin (KPSS) desenvolvido por Kwiatkowski et al. (1992). O teste ADF verifica se a série possui uma raiz unitária e possui as seguintes hipóteses: $\mathrm{H}_{0}$, a série possui uma raiz unitária, e $\mathrm{H}_{1}$, a série não possui uma raiz unitária (ENDERS, 2008). O teste KPSS testa a presença de raiz unitária na série pelas hipóteses $\mathrm{H}_{0}$, a série não possui uma raiz unitária, e $\mathrm{H}_{1}$, a série possui uma raiz unitária (BUENO, 2008). Adicionalmente, foram selecionadas automaticamente para o teste ADF zero defasagens para a série de Santarém e duas defasagens para a série de Belém com base no teste Scharwz Info Criterion. Para o teste KPSS foram selecionadas automaticamente pelo critério Newey-West Dandwith seis defasagens para a série de Santarém e três para a série de Belém. Os testes foram realizados para as séries dos dois municípios com a inclusão de um intercepto na equação de regressão. Os resultados dos testes mostraram que as séries são estacionárias ao nível de $1 \%$ de significância. 
Foi testada também a presença de efeito ARCH na variância das séries por meio do teste ARCH-LM desenvolvido por Engle (1982). O teste ARCH-LM analisa a hipótese nula de que não existe correlação entre os resíduos ao quadrado do modelo. O teste realizado utilizando os resíduos da equação de um modelo AR (1) em ambas as séries dos dois municípios apresentou $p$-valor inferior ao nível de $1 \%$ de significância, portanto aceitamos a hipótese de presença de efeito ARCH na variância da série.

Foram selecionadas apenas uma defasagem para os modelos, pois conforme acrescenta Silva (2016) em jogos repetidos de cartéis os agentes possuem informação suficiente para detectar e punir violações nos acordos estabelecidos. Isto, portanto, justifica neste trabalho a opção pela escolha de poucas defasagens nos modelos dado que os agentes possuem reação imediata as ações dos demais.

Os resíduos dos modelos se comportaram de maneira adequada e não há indícios de autocorrelação entre os resíduos dos modelos dos dois municípios. A hipótese de normalidade dos resíduos também foi atendida dada a suposição do Teorema Central do Limite, posto que a amostra dos períodos, presente na Tabela 1, possui extensão suficiente para prover a normalidade assintótica dos resíduos. Somado a isto, os testes de heterocedasticidade nos resíduos dos modelos não apresentaram indícios da presença de heterocedasticidade.

Com a verificação dos pré-requisitos de estacionariedade e heterocedasticidade verificados, foi realizada a estimação dos modelos para o município de Belém. A Tabela 3 apresenta os resultados dos modelos ARCH, GARCH, EGARCH e TGARCH para a média dos retornos dos preços da gasolina $\mathrm{C}$ do município de Belém.

Tabela 3. Resultados dos modelos para a média dos retornos de Belém.

\begin{tabular}{cllll}
\hline Coeficientes & ARCH (1) & GARCH $(1,1)$ & EGARCH $(1,1)$ & TGARCH $(1,1)$ \\
\hline$\varphi$ & 0,0000758 & 0,0000873 & $-0,0000499$ & 0,0000111 \\
& $(0,000195)$ & $(0,00018)$ & $(0,00023)$ & $(0,0002)$ \\
\hline$\theta_{1}$ & $-0,72076^{* * *}$ & $-0,53033^{* * *}$ & $-0,6458^{* * *}$ & $-0,54869^{* * *}$ \\
& $(0,0520)$ & $(0,0304)$ & $(0,0428)$ & $(0,0278)$ \\
\hline
\end{tabular}

Nível de significância: $* * * 1 \%, * * 5 \%, * 10 \%$. Os valores entre parênteses abaixo de cada coeficiente são os seus respectivos erros-padrão. Fonte: Autores.

Para a equação da média do modelo ARCH e todos os demais modelos foi utilizado um processo AR(1). A equação para a média dos retornos dos modelos para Belém apresentou valor positivo para o coeficiente da variável dummy, a única exceção foi o modelo EGARCH que apresentou valor negativo para o coeficiente da mesma variável. Estes resultados, entretanto, não foram confirmados em nenhum dos níveis de significância estabelecidos.

A Tabela 4 apresenta os resultados dos modelos ARCH, GARCH, EGARCH e TGARCH para variância dos retornos da gasolina $\mathrm{C}$ do município de Belém. A equação da variância do Modelo ARCH para Belém apresentou todos os coeficientes significativos. $\mathrm{O}$ coeficiente $\beta_{1}$ foi significativo e respeitou a condição de estabilidade. O coeficiente da variável dummy foi significativo e mostrou valor negativo. Assim, os resultados sugerem que há acúmulos de variância na série de Belém, seguidos por uma rigidez nos retornos evidenciada pelo coeficiente da variável dummy.

Os resultados da equação da variância do modelo GARCH também foram todos os coeficientes significativos. O modelo de Belém indicou pelo coeficiente da variável dummy que houve redução na variância da série durante o período de cartel, entretanto os coeficientes GARCH não respeitaram a condição de estabilidade $\alpha_{1}+\beta_{1}<1$, impossibilitando a determinação do modelo como adequado para modelar o comportamento da variância da série. 
Tabela 4. Resultados dos modelos para a variância de Belém.

\begin{tabular}{|c|c|c|c|c|}
\hline Coeficientes & $\mathrm{ARCH}(1)$ & GARCH $(1,1)$ & EGARCH $(1,1)$ & TGARCH $(1,1)$ \\
\hline$\alpha_{0}$ & $\begin{array}{l}0,0000394 * * * \\
(0,00000598)\end{array}$ & $\begin{array}{l}0,0000873 * * * \\
(0,00000316)\end{array}$ & $\begin{array}{l}-2,9350 * * * \\
(0,3028)\end{array}$ & $\begin{array}{l}0,0000125 * * * \\
(0,000004)\end{array}$ \\
\hline$\beta_{1}$ & $\begin{array}{l}0,9396 * * * \\
(0,1666)\end{array}$ & $\begin{array}{l}1,0982 * * * \\
(0,2185)\end{array}$ & $\begin{array}{l}0,9818 * * * \\
(0,1421)\end{array}$ & $\begin{array}{l}0,9392 * * * \\
(0,1994)\end{array}$ \\
\hline$\varphi$ & $\begin{array}{l}-0,0000150 * * \\
(0,00000634)\end{array}$ & $\begin{array}{l}-0,00000999 * * * \\
(0,00000317)\end{array}$ & $\begin{array}{l}-0,1050 \\
(0,0673)\end{array}$ & $\begin{array}{l}0,00000969 * * \\
(0,000004)\end{array}$ \\
\hline$\alpha_{1}$ & - & $\begin{array}{l}0,3216 * * * \\
(0,0456)\end{array}$ & $\begin{array}{l}0,7650 * * * \\
(0,0306)\end{array}$ & $\begin{array}{l}0,2828 * * * \\
(0,0420)\end{array}$ \\
\hline$\lambda_{1}$ & - & - & $\begin{array}{l}0,3276 \\
(0,3619)\end{array}$ & $\begin{array}{l}0,7057 \\
(0,3978)\end{array}$ \\
\hline
\end{tabular}

Nível de significância: $* * * 1 \%, * * 5 \%, * 10 \%$. Os valores entre parênteses abaixo de cada coeficiente são os seus respectivos erros-padrão.

Para a equação da variância no modelo EGARCH, houve diferentes comportamentos entre os municípios tanto no coeficiente de assimetria quanto no coeficiente da variável dummy. No modelo de Belém houve significância nos coeficientes da variância do modelo havendo evidências de acúmulos de volatilidade na série. Entretanto, o coeficiente da variável dummy não foi significativo, assim como o coeficiente da assimetria do modelo. Consequentemente não é possível inferir que, primeiro, houve redução da variância dos retornos durante o período de cartel, e, segundo, que a série apresenta choques assimétricos que impactam a variância de forma significativa.

Na equação da variância do modelo TGARCH de Belém todos os coeficientes foram significativos. Dois elementos podem ser destacados no modelo, o coeficiente da variável dummy foi positivo e significativo, não correspondendo a hipótese de menor variância na série durante o período de cartel, e por último que o coeficiente de assimetria $\left(\lambda_{t}\right)$ não foi significativo, não sustentando a hipótese de que a variância da série é afetada por impactos de choques negativos dos retornos.

No município de Santarém, surgiram alguns resultados nos coeficientes das variáveis dummy de cartel que não corresponderam as hipóteses de maiores preços e menor variância. A Tabela 5 apresenta os resultados dos modelos ARCH, GARCH, EGARCH e TGARCH, para a média dos retornos da gasolina C do município de Santarém.

A equação para a média dos retornos nos modelos de Santarém apresentou valor positivo para o coeficiente da variável dummy nos modelos ARCH, GARCH e TGARCH, e valor negativo para o coeficiente do modelo EGARCH. Apesar dos valores positivos do coeficiente da variável de cartel nos três modelos, apenas nos modelos ARCH e TGARCH houve significância. O coeficiente no modelo EGARCH não foi significante.

Tabela 5. Resultados dos modelos para a média dos retornos de Santarém.

\begin{tabular}{cllll}
\hline Coeficientes & ARCH $(1)$ & GARCH $(1,1)$ & EGARCH $(1,1)$ & TGARCH $(1,1)$ \\
\hline$\varphi$ & $0,000940^{* * *}$ & 0,00131 & $-0,0000541$ & $0,000725^{*}$ \\
& $(0,0089)$ & $(0,1189)$ & $(0,1113)$ & $(0,0419)$ \\
\hline$\theta_{1}$ & $-0,2253 * * *$ & 0,0538 & 0,1243 & $-0,2390 * * *$ \\
& $(0,0000247)$ & $(0,0008)$ & $(0,0008)$ & $(0,0004)$ \\
\hline
\end{tabular}

Nível de significância: $* * * 1 \%, * * 5 \%, * 10 \%$. Os valores entre parênteses abaixo de cada coeficiente são os seus respectivos erros-padrão. Fonte: Autores. 
A Tabela 6 por sua vez descreve os resultados dos modelos estimados para a variância do município de Santarém. Dessa forma, equação da variância do modelo ARCH para o município de Santarém apresentou todos os coeficientes significativos. O coeficiente $\beta_{1}$ do termo ARCH no modelo de Santarém foi significativo, porém não respeitou a condição de estabilidade. Assim, embora haja evidências de que há redução da variância conforme apontado pelo coeficiente da variável dummy, não é possível extrair maiores conclusões do modelo dada a condição verificada no coeficiente.

A modelagem por meio do modelo GARCH para Santarém também apresentou todos os coeficientes significativos. O modelo exibiu os coeficientes de modelagem da variância dentro das condições de estabilidade e o coeficiente da variável dummy de cartel foi negativo conforme esperado, indicando a redução na variância da série no período de cartel.

Conforme observado na equação da variância no modelo EGARCH de Belém, houve diferentes comportamentos tanto no coeficiente de assimetria quanto no coeficiente da variável dummy de cartel. O modelo EGARCH de Santarém apresentou coeficientes significativos para a modelagem da variância indicando que há acúmulos de volatilidade na série e condicionalidade da variância com períodos anteriores. O coeficiente da variável dummy foi significativo, contudo, não satisfez a hipótese de redução da variância no período de cartel estabelecido. Por último, o coeficiente de assimetria do modelo não foi significativo, sugerindo a rejeição da hipótese de que a variância da série é afetada em maior grau por impactos de choques negativos dos retornos.

Na equação da variância do modelo TGARCH de Santarém, foi verificado que o coeficiente da variável dummy de cartel foi positivo, entretanto não foi significativo. Além disso, o coeficiente de assimetria foi positivo e significativo, simbolizando que a série possui impacto de choques assimétricos na variância.

Tabela 6. Resultados dos modelos para a variância de Santarém.

\begin{tabular}{cllll}
\hline Coeficientes & ARCH $(1)$ & GARCH $(1,1)$ & EGARCH $(1,1)$ & TGARCH $(1,1)$ \\
\hline$\alpha_{0}$ & $0,0000296^{* * *}$ & $0,0000162 * * *$ & $-4.2346^{* * *}$ & $0,00000192 * * *$ \\
& $(0,0000432)$ & $(0,000005)$ & $(0,4851)$ & $(0,0000003)$ \\
\hline$\beta_{1}$ & $\begin{array}{l}11.513 * * * \\
(0,4420)\end{array}$ & $\begin{array}{l}0,1046^{*} \\
(0,0567)\end{array}$ & $\begin{array}{l}0,4905 * * * \\
(0,0682)\end{array}$ & $\begin{array}{l}0,0102 * * \\
(0,0049)\end{array}$ \\
\hline$\varphi$ & - & $0,00000530 * * *$ & $0,1580 * * *$ & 0,0000000470 \\
& $0,0000279 * * *$ & $(0,0000017)$ & $(0,0401)$ & $(0,0000005)$ \\
\hline \multirow{2}{*}{$\alpha_{1}$} & $(0,00000429)$ & $0,6458 * * *$ & $0,5898 * * *$ & $0,9139 * * *$ \\
& - & $(0,1117)$ & $(0,0478)$ & $(0,0109)$ \\
\hline$\lambda_{1}$ & - & - & $-0,0724$ & $0,4254 * * *$ \\
& - & - & $(0,0568)$ & $(0,0837)$ \\
\hline
\end{tabular}

Nível de significância: $* * * 1 \%, * * 5 \%, * 10 \%$. Os valores entre parênteses abaixo de cada coeficiente são os seus respectivos erros-padrão. Fonte: Autores.

Nas equações da média dos dois municípios há um fato interessante de se notar. Em ambos os municípios houve diferentes resultados para o coeficiente da variável dummy de cartel na equação da média, sugerindo que a hipótese de maiores preços durante o período de cartel não é razoável para os dois municípios, não representando, portanto, indícios de cartel nos períodos estabelecidos. Como apontado por Vasconcelos e Vasconcelos (2008) aumentos de preços não são condição necessária para que possa ser determinado a presença de um cartel, pois ao se observar apenas os preços não é possível inferir se tais resultados são derivados de um acordo de maximização conjunta de lucros (cartel) ou de um equilíbrio de Nash.

É notável que houve comportamento diferente do esperado na variável dummy de cartel para a média, na qual esperava-se coeficientes positivos e significativos para o período de cartel, o que não aconteceu. Entretanto, mesmo com 
comportamento distante do esperado na equação para a média, os modelos ainda sim seguiram a hipótese de menor variância no período de cartel, como o modelo ARCH em Belém, constituindo indícios de um possível cartel em ambos os períodos. De acordo com Bolotova et al. (2008) é possível que haja redução na variância dos preços sem que seja simultaneamente verificado um aumento nos preços. Isto pode ocorrer dada a estratégia das firmas de buscarem extrair lucros por meio da homogeneização dos seus preços, ao invés de aumentá-los. Dessa forma, tal fato qualifica a variância como principal variável a se observar em casos de cartel.

A sinalização da presença de choques assimétricos pelo coeficiente de assimetria na variância da série de Santarém parece sugerir um comportamento ambíguo. Se de um lado no modelo EGARCH não há a confirmação da hipótese da presença de choques assimétricos na variância da série, por outro o modelo TGARCH confirmou esta hipótese com o coeficiente positivo e significativo. Conforme apresenta Silveira et al. (2019) esta situação poderia ser entendida a partir da perspectiva do comportamento das firmas sob um cartel, pois os períodos de menor variância (cartel) são os períodos em que há estabilidade no acordo entre as firmas, não havendo, portanto, dissidentes do cartel. Noutro cenário, se caso estivéssemos diante de um período de guerra de preços e punições a dissidentes do cartel, a variância da série poderia apresentar comportamento instável e semelhante ao de um período competitivo, o que poderia justificar a interpretação ambígua do coeficiente de assimetria nos dois modelos. Outrossim, estes dois modelos não confirmaram a hipótese de menor variância no período de cartel, não apresentando indícios de acordos entre as firmas em ambos os períodos.

\section{Considerações Finais}

Pelo que foi visto até este ponto neste estudo e na literatura acerca do tema, os modelos são capazes de modelar tanto a média dos preços dos combustíveis quanto a sua variância. Os modelos EGARCH e TGARCH mesmo ainda com pouca presença na literatura acerca de métodos de detecção de cartéis, também podem ser utilizados como ferramenta para este fim. Ademais, estes dois modelos viabilizam a verificação da presença de choques assimétricos nas séries de preços, o que os torna mais efetivos e aumenta a sua capacidade de modelagem das variáveis para detecção de cartéis.

Pelo que foi demonstrado nos modelos EGARCH e TGARCH, o comportamento do coeficiente de assimetria nos dois modelos poderia ser esperado, posto que grande parte das séries de preços de combustíveis não exibem comportamentos muito voláteis quando há choques negativos derivados dos preços de distribuição ou de outros custos. Diferentemente, conforme aponta Salvini et al. (2017), as séries de combustíveis apresentam comportamentos distintos quanto a transmissão de preços ao consumidor, visto que elevações (choques positivos) tendem a serem repassadas de forma assimétrica no curto prazo com relação às reduções nos preços de distribuição (custos).

Com relação às equações para a média nos modelos, convém ressaltar que embora seja esperado pela intuição que haja aumentos de preços durante um cartel, não necessariamente isto se verifica na realidade, pois elevações dos preços não são condição necessária nem suficiente para que haja presença de cartel. Com isso não descartamos a possibilidade de que haja de fato um cartel, mesmo que não haja indícios na média dos preços. Pode-se levantar também a hipótese de que caso o período de cartel selecionado contenha os resultados de períodos de guerras de preços entre as firmas, este fato seria refletido justamente nos valores do coeficiente da variável dummy de cartel, não indicando elevações nos preços entre as firmas.

O propósito de trabalhos como este, para além de fornecer ferramentas para detecção de cartéis, é esclarecer sobretudo o que não são cartéis. Pois se as ferramentas são direcionadas para onde há maior probabilidade de existir um acordo entre firmas para burlar a concorrência, maiores serão as chances de êxito da autoridade legal nas investigações para a detecção do acordo. Este entendimento é bastante adequado para superar o senso comum e apresentar evidências acerca de cartéis entre firmas, especialmente entre postos de combustíveis. 
As hipóteses estabelecidas não foram atendidas em alguns modelos como pôde ser visto ao longo da análise dos resultados. Isto não necessariamente representa uma falha na metodologia, ou tampouco determina a não existência de carteis. É razoável admitir que a trajetória da ciência não é feita só de hipóteses correspondidas, e o esforço empenhado neste trabalho esteve a todo instante sujeito a possibilidade de não ter suas hipóteses atendidas.

Diante de tudo que foi exposto acerca das ferramentas e métodos de detecção de cartéis, sugere-se que estes mesmos modelos sejam abordados com o uso de ferramentas de machine learning para entender as dinâmicas de preços entre os postos para facilitar o processo de deteç̧ão de acordos de cartel. Recomenda-se também que sejam realizados estudos com machine learning para os preços de postos em diferentes regiões geográficas em uma mesma cidade ou região para facilitar o processo de análise e detecção de possíveis acordos entre os postos.

\section{Referências}

Abrantes-Metz, R. M., Froeb, L. M., Geweke, J., \& Taylor, C. T. (2006). A variance screen for collusion. International Journal of Industrial Organization, 24(3), 467-486.

Abrantes-Metz, R., \& Bajari, P. (2009). Screen for conspiracies and their multiple applications. Antitrust, $24,66$.

ANP - Agência Nacional do Petróleo, Gás Natural e BIOCOMBUSTÍVEIS. Anuário estatístico brasileiro do petróleo, gás natural e biocombustíveis: 2020. http://www.anp.gov.br/publicacoes/anuario-estatistico/5809-anuario-estatistico-2020.

Bolotova, Y., Connor, J. M., \& Miller, D. J. (2008). The impact of collusion on price behavior: Empirical results from two recent cases. International Journal of Industrial Organization, 26(6), 1290-1307.

Bollerslev, T. (1986). Generalized autoregressive conditional heteroskedasticity. Journal of econometrics, 31(3), 307-327.

Bueno, R. L. (2008). Econometria de séries temporais. Cengage Learning.

Buccirossi, P. (2006). Does parallel behavior provide some evidence of collusion? Review of Law \& Economics, 2(1), 85-102.

BRASIL. Lei $n^{\circ}$ 8.137, de 27 de dezembro de 1990. Define crimes contra a ordem tributária, econômica e contra as relações de consumo, e dá outras providências. Presidência da República, 1990. http://www.planalto.gov.br/ccivil_03/LEIS/L8137.htm.

Carrijo, G. D. (2019). Análise estatística dos preços de combustíveis para auxílio na detecção de cartéis no setor de distribuição. http://repositorio.enap.gov.br/handle/1/4111.

Conselho Administrativo De Defesa Econômica. (2009). Coleção SDE/CADE no 01/2009: Combate a cartéis e programa de leniência. Diário Oficial da União, Brasília, DF, $3^{\text {a }}$ edição, 36 páginas, 2009. http://www.cade.gov.br/acesso-a-informacao/publicacoes-institucionais/documentos-da-antigalei/cartilha_leniencia.pdf.

Dickey, D. A., \& Fuller, W. A. (1979). Distribution of the estimators for autoregressive time series with a unit root. Journal of the American statistical association, 74(366a), 427-431.

Enders, W. (2008). Applied econometric time series. John Wiley \& Sons.

Engle, R. F. (1982). Autoregressive conditional heteroscedasticity with estimates of the variance of United Kingdom inflation. Econometrica: Journal of the econometric society, $987-1007$.

Glosten, L. R., Jagannathan, R., \& Runkle, D. E. (1993). On the relation between the expected value and the volatility of the nominal excess return on stocks. The journal of finance, 48(5), 1779-1801.

Greene, W. H. (2000). Econometric analysis. 4th edition. International edition, Prentice Hall, 201-215.

Grout, P. A., \& Sonderegger, S. M. I. A. (2007). Structural approaches to cartel detection. In European Competition Law Annual: 2006. Enforcement of Prohibition of Cartels (pp. 83-103). Hart Publishing.

Harrington, J. E. (2005). Detecting cartels. N. 526. working paper.

Harrington, J., 2006. Behavioral screening and the detection of cartels. In: ClausDieter, E., Atanasiu, I. (Eds.), European Competition Law Annual 2006: Enforcement of Prohibition of Cartels. Hart Publishing, Oxford, UK.

Harrington, J. E. (2012). A theory of tacit collusion N. 588. Working Paper.

Harrington Jr, J. E. (2021). The Practical Requirements of a Successful Cartel.

Instituto Brasileiro De Geografia E Estatística. Frota de veículos, 2021. https://cidades.ibge.gov.br/brasil/pa/pesquisa/22/28120.

Kwiatkowski, D., Phillips, P. C., Schmidt, P., \& Shin, Y. (1992). Testing the null hypothesis of stationarity against the alternative of a unit root: How sure are we that economic time series have a unit root? Journal of econometrics, 54(1-3), 159-178. 
Research, Society and Development, v. 10, n. 13, e279101321397, 2021

(CC BY 4.0) | ISSN 2525-3409 | DOI: http://dx.doi.org/10.33448/rsd-v10i13.21397

Marques, M. I. G. (2017). Aplicação dos modelos GARCH, EGARCH e TGARCH no DAX-30. Dissertação de Mestrado, Instituto Politécnico de Lisboa, Portugal. http://hdl.handle.net/10400.21/7211

Morettin, P. A. (2017). Econometria financeira: um curso em séries temporais financeiras. Editora Blucher.

Pedra, D. P., de Oliveira Bicalho, L. M. N., de Araújo Vilela, O., Baran, P. H., de Paiva, R. M., \& de Melo, T. P. (2010). Metodologia adotada pela agência nacional do petróleo, gás natural e biocombustíveis para a detecção de cartéis. ANP, Rio de Janeiro.

Ragazzo, C. E. J., \& Silva, R. D. (2006). Aspectos econômicos e jurídicos sobre cartéis na revenda de combustíveis: uma agenda para investigações. SEAE/MF Documento de trabalho, 40 .

Robinson, J. (1969). The economics of imperfect competition. Springer.

Silva, A. S. (2016). Filtros de cartéis baseados em dinâmicas de preços: uma aplicação ao varejo de combustíveis do Brasil. 224 folhas. Tese (Doutorado) UFJF, Programa de Pós-graduação em Economia, Juiz de Fora, Brasil.

Salvini, R. R., Burnquist, H. L., \& Jacomini, R. L. (2017). Investigando a assimetria na transmissão dos preços dos combustíveis no Estado de São Paulo. Anais do Seminário Científico do UNIFACIG, (2).

Silveira, D., Vasconcelos, S., Bogossian, P., \& Neto, J. (2021). Cartel screening in the Brazilian fuel retail market. EconomiA, 22(1), 53-70.

Vasconcelos, S. P., \& Vasconcelos, C. F. (2008). Análise do comportamento estratégico em preços no mercado de gasolina brasileiro: modelando volatilidade. Análise Econômica, 26(50).

Zakoian, J. M. (1994). Threshold heteroskedastic models. Journal of Economic Dynamics and control, 18(5), 931-955. 